\title{
Glycyrrhizic acid promotes osteogenic differentiation of human bone mesenchymal stem cells by activating the Wnt/ $\beta$-catenin signalling pathway
}

\author{
Deting Xue ${ }^{1}$, Jinwu Bai $^{1}$, Jianxiang Xu${ }^{1}$, Kai Hang ${ }^{1}$, Zhihui Kuang ${ }^{1}$, Chenwei Zhou ${ }^{1}$, Li \\ Ying $^{1}$, and Yibo Wang ${ }^{1}$ \\ ${ }^{1}$ Zhejiang University School of Medicine Second Affiliated Hospital
}

July 28, 2020

\begin{abstract}
Background and Purpose Glycyrrhizic acid (GA) is a major triterpene glycoside isolated from liquorice root that has been shown to inhibit osteoclastogenesis. However, there have been no reports regarding the effect of GA on osteogenic differentiation. Therefore, this study was performed to explore the effects and mechanism of action of GA on osteogenesis. Experimental Approach A CCK-8 array was used to assess cell viability. The osteogenic capability was investigated by real-time quantitative PCR, western blotting and immunofluorescence analyses. ALP staining and ARS were used to evaluate ALP activity and mineralisation, respectively. GA-GelMA hydrogels were designed to verify the therapeutic effects of GA in vivo by radiographic analysis and histological evaluation. Key Results Our results show that GA had no significant influence on the viability or proliferation of human bone mesenchymal stem cells (hBMSCs). GA promoted osteogenic differentiation and enhanced calcium deposition. Furthermore, levels of active $\beta$-catenin protein increased after treatment $\mathrm{w}$ ith GA. Wnt/catenin signalling inhibitor partially reversed the effects of GA on osteogenic differentiation. In a mouse femoral fracture model, GA-GelMA hydrogels accelerated bone healing. Conclusion and Implications Our results show that GA promotes the osteogenic differentiation of hBMSCs by modulating the Wnt/ $\beta$-catenin signalling pathway. GA-GelMA hydrogels promoted bone fracture healing. GA has potential as a cost-effective treatment of bone defects.
\end{abstract}

Title:Glycyrrhizic acid promotes osteogenic differentiation of human bone mesenchymal stem cells by activating the $\mathrm{Wnt} / \beta$-catenin signalling pathway

Running title: Glycyrrhizic acid promotes osteogenesis through Wnt/ $\beta$-catenin pathway

Authorship: Jinwu Bai ${ }^{1,2,+}$, Jianxiang $\mathrm{Xu}^{1,2,+}$, Kai Hang ${ }^{1,2,+}$, Zhihui Kuang ${ }^{1,2}$, Li Ying ${ }^{1,2}$, Chenwei Zhou ${ }^{1,2}$, Licheng $\mathrm{Ni}^{1,2}$, Yibo Wang ${ }^{1,2}$, Deting Xue ${ }^{1,2,{ }^{*}}$

${ }^{1}$ Department of Orthopaedics, The Second Affiliated Hospital, Zhejiang University School of Medicine, Zhejiang University, No.88, Jiefang Road, Hangzhou 310009, People's Republic of China

${ }^{2}$ Orthopedics Research Institute of Zhejiang University. No.88, Jiefang Road, Hangzhou 310009, People's Republic of China

+These authors contributed equally to this work and should be considered as co-first authors.

*Corresponding author:

Deting Xue M.D. Department of Orthopaedics, the Second Affiliated Hospital, School of Medicine, Zhejiang University, No. 88 Jie Fang Road, Hangzhou 310009, Zhejiang, China

E-mail address:blueskine@zju.edu.cn 
Fax: 0086-571-87022776; Tel: +86571 8778 3530;

Word Count (excluding figure legends and references)

Abstract: 212 words

Methods: 1931 words

Discussion and Conclusions: 944 words

\section{Conflict of Interest Statement:}

The authors, their immediate families, and any research foundations with which they are affiliated have not received any financial payments or other benefits from any commercial entity related to the subject of this article. None of the authors have any conflicts of interest with regards to this research.

\section{Ethical Review Committee:}

All animal experiments were conducted in accordance with principles and procedures approved by the Institutional Animal Care Use Committee at the second affiliated hospital of Zhejiang University (approval number: 2020-No.25) All of the treatment procedures conformed to the ethical standards established in the 1964 Declaration of Helsinki.

\section{Bullet point summary}

\section{- What is already known}

GA has been reported to have a variety of pharmacological activities, including anti-viral, anti-tumour, anti-inflammatory and anti-oxidative activities. GA effectively inhibited osteoclast maturation and bone resorption and exhibited an osteoprotective effect in ovariectomy mice.

\section{-What this study adds}

This study was performed to explore the effects and mechanism of action of GA on osteogenesis. In the meantime, we designed a GA-GelMA that used for the mice femur fracture healing.

\section{-Clinical significance}

Our study further enriched the pharmacological action and potential application of GA. GA has potential as a cost-effective treatment of bone fracture or defects.

\section{Abstract}

\section{Background and Purpose}

Glycyrrhizic acid (GA) is a major triterpene glycoside isolated from liquorice root that has been shown to inhibit osteoclastogenesis. However, there have been no reports regarding the effect of GA on osteogenic differentiation. Therefore, this study was performed to explore the effects and mechanism of action of GA on osteogenesis.

\section{Experimental Approach}

A CCK-8 array was used to assess cell viability. The osteogenic capability was investigated by real-time quantitative PCR, western blotting and immunofluorescence analyses. ALP staining and ARS were used to evaluate ALP activity and mineralisation, respectively. GA-GelMA hydrogels were designed to verify the therapeutic effects of GA in vivo by radiographic analysis and histological evaluation.

\section{Key Results}

Our results show that GA had no significant influence on the viability or proliferation of human bone mesenchymal stem cells (hBMSCs). GA promoted osteogenic differentiation and enhanced calcium deposition. Furthermore, levels of active $\beta$-catenin protein increased after treatment w ith GA. Wnt/catenin signalling 
inhibitor partially reversed the effects of GA on osteogenic differentiation. In a mouse femoral fracture model, GA-GelMA hydrogels accelerated bone healing.

\section{Conclusion and Implications}

Our results show that GA promotes the osteogenic differentiation of hBMSCs by modulating the Wnt/ $\beta$ catenin signalling pathway. GA-GelMA hydrogels promoted bone fracture healing. GA has potential as a cost-effective treatment of bone defects.

\section{Key words}

glycyrrhizic acid, human bone mesenchymal stem cells (hBMSCs), osteogenesis, Wnt/ $\beta$-catenin

\section{Abbreviations}

GelMA hydrogels, Gelatin Meth-acryloyl hydrogels; BMP, bone morphogenetic protein; RANKL, receptor activator of nuclear factor $x \mathrm{~B}$ ligand; ALP, Alkaline phosphatase; ARS, Alizarin red staining; GAPDH, glyceraldehyde-3-phosphate dehydrogenase; COL1A1, collagen type I a 1chain; RUNX2, runt-related transcription factor 2; OCN, osteocalcin; active $\beta$-catenin, non-phosphorylated (active) $\beta$-catenin; t- $\beta$-catenin, total $\beta$-catenin; RT-PCR, BV/TV, trabecular bone volume per total volume; Tb.Th, trabecular bone thickness; Tb.N, trabecular bone number; Tb.Sp, trabecular bone separation; H\&E, haematoxylin and eosin. bone tissue engineering (BTE). mitogen-activated protein kinase (MAPK). extracellular regulated protein kinases (ERK). phosphatidylinositol 3 kinase (PI3K). protein kinase

$\mathrm{B}(\mathrm{AKT})$.

\section{Introduction}

Bone defects or fracture non-union caused by high-energy injury, infection and bone tumours are common clinical problems in orthopaedic and trauma surgery (Dimitriou et al., 2011; Fazzalari, 2011; Giannoudis et al., 2011). The incidence of bone non-union has been estimated to be 5-10\% (Zura et al., 2016). Bone marrow-derived mesenchymal stem cells (BMSCs) have self-renewal capabilities and are able to differentiate into a variety of cell types, including osteoblasts, chondrocytes, adipocytes and myocytes (Ankrum et al., 2014). It is vital to promote the recruitment of mesenchymal stem cells (MSCs) in the microenvironment surrounding a fracture defect and to induce differentiation of MSCs into osteoblasts to facilitate bone healing (Coutu et al., 2017; Gibon et al., 2016; Park et al., 2015).

Many strategies are used to promote bone healing. Bone autografting is the gold standard treatment for nonunion. However, autografting has a number of limitations, including limited availability, donor site morbidity and severe complications (Dekker et al., 2017). BMP-2 and BMP-7 are considered essential in bone healing, and have been approved for clinical use (Begam et al., 2017; Fassbender et al., 2014). Nevertheless, the high cost of these agents has limited their clinical application. Another promising method is gene therapy involving the transfer of target genes that strongly promote osteogenesis into the host genome. However, gene therapy is also associated with a number of problems, including high cost and concerns over biological safety (Atasoy-Zeybek et al., 2018; Shapiro et al., 2018). Therefore, it is important to find a cost-effective and safe strategy to remedy bone defects.

Many small molecular compounds derived from natural products, such as Chinese herbs, are abundant and inexpensive, with good pharmacological benefits and potential for clinical application. Liquorice is an extremely important Chinese herbal medicine that has been widely used for millennia due to its palatable taste and medicinal potential. Glycyrrhizic acid (GA) is a major triterpene glycoside isolated from liquorice root (Feng et al., 2015; Sun et al., 2019), and has been reported to have a variety of pharmacological activities, including anti-viral, anti-tumour, anti-inflammatory and anti-oxidative activities (Bhattacharjee et al., 2012; Su et al., 2017; Sun et al., 2019; Wang et al., 2011). However, there has been little research regarding use of GA in bone regeneration. Yin et al. (Yin et al., 2019) reported that GA effectively inhibited osteoclast maturation and bone resorption and exhibited an osteoprotective effect in ovariectomy mice. $\mathrm{Li}$ et al. (Li et al., 2018) reported that GA inhibited RANKL-induced osteoclastogenesis. The effects of GA 
in osteoclastogenesis have been explored. However, there have been no studies regarding the influence of GA on osteogenesis. Therefore, this study was performed to explore the effects of GA on the proliferation and osteogenic differentiation of human bone mesenchymal stem cells (hBMSCs) as well as the underlying mechanisms.

\section{Materials and Methods}

\section{Reagents}

GA (purity, $>97.5 \%$, as determined by high-performance liquid chromatography [HPLC]) was purchased from TargetMol (Wellesley Hills, MA, USA). The GA was dissolved in dimethyl sulfoxide (DMSO) and stored at $-20^{\circ} \mathrm{C}$ in the dark. Photopolymerisable gelatine methacryloyl (GelMA) hydrogels were purchased from Engineering For Life (Suzhou, China). XAV-939, an inhibitor of the Wnt/ $\beta$-catenin signalling pathway, was purchased from Selleck Chemicals (Houston, TX, USA). We used $10 \mu \mathrm{M}$ XAV-939 based on a previous study (Iwatani et al., 2017).

Cell isolation and culture

hBMSCs were isolated from the whole bone marrow of two healthy donors (two men, aged 20 and 22 years) using the method described previously (Zhang et al., 2018). All donors provided informed consent before collection of their bone marrow and the protocol was approved by the Ethics Committee of the Second Affiliate Hospital of Zhejiang University. All of the treatment procedures conformed to the ethical standards of the Declaration of Helsinki. Adherent hBMSCs were then cultured in culture flasks with hBMSC growth medium (Cyagen Biosciences, Guangzhou, China) in an incubator at $37^{\circ} \mathrm{C}$ with $5 \% \mathrm{CO}_{2}$, with passage after reaching $80 \%$ confluence. Cells from passages $3-5$ were used in subsequent experiments.

Cell viability assay

To assess the effects of GA on the proliferation of hBMSCs, cells were seeded into 96-well plates (5,000/well) and allowed to adhere for 12 hours. The medium was then changed, and the cells were subsequently treated with various concentrations of GA $(0,1,10,100 \mu \mathrm{M})$ for 1,2 or 3 days. The medium was then changed to $10 \%$ Cell Counting Kit-8 (CCK-8; Dojindo, Kumamoto, Japan) in $100 \mu \mathrm{L}$ of low-sugar Dulbecco's modified Eagle's medium (L-DMEM) without foetal bovine serum (FBS) (Gibco, Waltham, MA, USA) for 4 hours at $37^{\circ} \mathrm{C}$. The absorbance at $450 \mathrm{~nm}\left(\mathrm{~A}_{450}\right)$, which is proportional to cell proliferation, was measured using a microplate reader (ELX808; BioTek, Winooski, VT, USA).

Osteogenic differentiation protocol

Osteogenesis of hBMSCs was induced by osteogenic induction medium (OIM) consisting of L-DMEM, 10\% FBS, $100 \mathrm{IU} / \mathrm{mL}$ penicillin/streptomycin, $100 \mathrm{nM}$ dexamethasone, $0.2 \mathrm{mM}$ ascorbic acid and $10 \mathrm{mM} \beta-$ glycerophosphate. Cells were cultured in growth medium (L-DMEM; $10 \%$ FBS and $100 \mathrm{IU} / \mathrm{mL}$ penicillin/streptomycin) in 6- or 12-well cell culture plates at a density of $3 \times 10^{4} / \mathrm{cm}^{2}$ and incubated at $37 \mathrm{degC}$ in an atmosphere of $5 \% \mathrm{CO}_{2}$. After cells reached about $80-90 \%$ confluence, the culture medium was aspirated off and replaced with fresh OIM. The cells were maintained by the addition of fresh OIM every 2-days. In addition, GA was added to the OIM at concentrations ranging from 0.01 to $100 \mu \mathrm{M}$.

ALP staining and ALP activity assay

To investigate early mineralisation, hBMSCs were treated with OIM and GA at different concentrations in 12 -well plates for 3 days. For ALP staining, cells were fixed with $4 \%$ paraformaldehyde (Sangon Biotech, Shanghai, China) for 30 minutes. Cells were then washed with double distilled water $\left(\mathrm{ddH}_{2} \mathrm{O}\right)$ three times and stained using an Alkaline Phosphatase Color Development Kit (Beyotime, Shanghai, China). For measurement of ALP activity, cells were lysed with lysis buffer consisting of $20 \mathrm{mM}$ Tris-HCl (pH 7.5), $150 \mathrm{mM}$ $\mathrm{NaCl}$ and $1 \%$ Triton X-100. ALP activity was determined by the ALP Activity Assay (Beyotime) according to the manufacturer's instructions. Finally, the conversion colour of $p$-nitrophenyl phosphate was measured after 3 days of culture in osteogenic medium at 405/650 nm. 
Alizarin red staining and quantification assay

Alizarin red staining (ARS; Cyagen Biosciences) was performed to assess late mineralisation. hBMSCs were seeded in 12-well plates and cultured with OIM and GA at various concentrations. After 10 days of culture, cells were fixed in $4 \%$ paraformaldehyde for 20 minutes at room temperature and subsequently washed three times with $\mathrm{ddH}_{2} \mathrm{O}$. Finally, the cells were treated with ARS $(0.5 \%, \mathrm{pH} 4.1-4.2)$ for 20 minutes and then rinsed with distilled water. To quantify the staining, stained mineralised nodules were desorbed with $10 \%$ cetylpyridinium chloride (Sigma, Shanghai, China) and the $\mathrm{A}_{570}$ was measured.

Western blotting analysis

Cells were lysed in RIPA buffer supplemented with proteasome and phosphatase inhibitors (Beyotime). Equal amounts of proteins were separated by 10\% SDS-PAGE and then transferred onto polyvinylidene fluoride (PVDF) membranes (Millipore, Shanghai, China). After blocking in 5\% non-fat milk for 1 hour, the membranes were incubated overnight at $4^{\circ} \mathrm{C}$ with antibodies specific to GAPDH $(1: 1,500$; Cell Signaling Technology, Danvers, MA, USA), COL1A1 (1:1,000; Abcam, Cambridge, UK), RUNX2 (1:1,600; Abcam), active $\beta$-catenin $(1: 1,000$; Abcam, Shanghai, China) or total $\beta$-catenin $(1: 1,000 ;$ Abcam). After washing four times ( 5 minutes each time) in Tris-buffered saline with $0.1 \%$ Tween 20 (TBST), the membranes were incubated with horseradish peroxidase-conjugated secondary anti-mouse or anti-rabbit antibodies (Beyotime) for 1 hour at room temperature. After washing three times ( 5 minutes each time) with TBST, proteins were detected using enhanced chemiluminescence blotting reagents (Millipore). Signal intensity was measured using a Bio-Rad XRS chemiluminescence detection system (Bio-Rad, Hercules, CA, USA).

Immunofluorescence assay

Cells were cultured in 12-well plates with OIM. After induction of osteogenesis, cells were fixed in $4 \%$ paraformaldehyde for 15 minutes at room temperature, permeabilised in $0.05 \%$ Triton X-100 for 30 minutes and blocked in $2 \%$ bovine serum albumin (BSA) for 30 minutes. Fixed cells were washed and incubated overnight with anti-RUNX2 (1:500; Abcam) or anti-COL1A1 (1:500; Abcam) antibody. The cells were then incubated with fluorescence-conjugated secondary antibody (DyLight 550 Conjugate; Boster Biological Technology, Wuhan, China) for 2 hours and nuclei were stained with 2-(4-amidinophenyl)-6-indolecarbamidine dihydrochloride (DAPI) (Beyotime) for 5 minutes. Samples were observed under a fluorescence microscope (EU5888; Leica, Wetzlar, Germany).

RNA isolation and real time quantitative PCR

Total RNA was isolated from cells cultured with OIM using RNAiso reagent (Takara Bio Inc., Dalian, China) and quantified by measuring the $\mathrm{A}_{260}$ (NanoDrop 2000; Thermo Fisher Scientific, Waltham, MA, USA). First strand cDNA was synthesised using PrimeScript RT Master Mix (Takara Bio Inc.) according to the manufacturer's instructions. Total RNA ([?] 1,000 ng) was reverse-transcribed into cDNA in a reaction volume of $20 \mu \mathrm{L}$ using a Double-Strand cDNA Synthesis Kit (Takara Bio Inc.). The levels of mRNAs encoding COL1A1, RUNX2, OCN, osterix, ALP and GAPDH were determined using a StepOnePlus real-time PCR system (Applied Biosystems Inc., Warrington, UK) and SYBR Premix Ex Taq (Takara Bio Inc.) under the following conditions: $95^{\circ} \mathrm{C}$ for $30 \mathrm{~s}$ followed by 40 cycles of $95^{\circ} \mathrm{C}$ for $5 \mathrm{~s}$ and $60^{\circ} \mathrm{C}$ for $30 \mathrm{~s}$. GAPDH was used as an internal control and allowed adjustment of differences among samples. DNA concentrations were calculated using the $2^{-\mathrm{Ct}}$ method (Livak et al., 2001). All primers used in this experiment were synthesised by Sangon Biotech and are listed in Table 1.

Fabrication of GelMA, GA/GelMA scaffolds and structural characterisation

Fabrication of GelMA was performed in accordance with the manufacturer's instructions. Briefly, the GelMA powder was dissolved in $0.25 \%(\mathrm{w} / \mathrm{v}$ ) lithium acylphosphinate salt (LAP) as a photoinitiator with or without GA. The hydrogel was then sterilised with a $0.22 \mu \mathrm{m}$ polyethersulfone (PES) membrane syringe filter (Guangzhou Jet Bio-Filtration, Guangzhou, China). The concentration of GelMA was 10\%, and the concentration of GA in the $10 \%$ GA/GelMA was $2 \mathrm{mM}$. Finally, the material was crosslinked using a $365-\mathrm{nm}$ ultraviolet (UV) lamp with a light intensity of $2.7 \mathrm{~mW} / \mathrm{cm}^{2}$ for $15 \mathrm{~s}$ and lyophilised for 24 hours. The surface 
structure of samples was examined by scanning electron microscopy (SEM) (FEI Q45; FEI Co., Portland, OR, USA).

Lentiviral packaging and cell infection

A lentiviral green fluorescent protein (GFP) vector was purchased from Cyagen Biosciences and transfections were performed according to the manufacturer's instructions. Briefly, hBMSCs were incubated with GFP lentiviral particles and $5 \mu \mathrm{g}$ polybrene/mL in growth medium. After 18 hours, the infection medium was replaced with hBMSC growth medium. After 2 days, the transfection results were observed by fluorescence microscopy (EU5888; Leica Camera, Wetzlar, Germany).

GFP-hBMSC 2D and 3D culture in GelMA

For 2D culture, 10\% GelMA was spread over 48-well plates and crosslinked using a 365-nm UV lamp. GFPhBMSCs were then seeded on the surface of GelMA and cultured in growth medium at $37^{\circ} \mathrm{C}$ in an atmosphere of $5 \% \mathrm{CO}_{2}$. For $3 \mathrm{D}$ culture, the cultured hBMSCs were trypsinised and collected by centrifugation. The resulting pellets were introduced onto $10 \%$ GelMA pre-solution and $0.25 \%(\mathrm{w} / \mathrm{v}) \mathrm{LAP}$ as a photoinitiator, and sterilised with a $0.22 \mu \mathrm{m}$ polyethersulfone (PES) membrane syringe filter (Guangzhou Jet Bio-Filtration). Finally, the material was crosslinked using a $365 \mathrm{~nm}$ UV lamp and washed twice before culturing the hydrogels in hBMSC growth medium at $37^{\circ} \mathrm{C}$ in an atmosphere of $5 \% \mathrm{CO}_{2}$.

In vivo evaluation in animals

All animal experiments were conducted in accordance with principles and procedures approved by the Institutional Animal Care Use Committee at the second affiliated hospital of Zhejiang University (approval number: 2020-No.25). A mouse femoral fracture model was created as described previously (Johnson et al., 2018; Murata et al., 2014) in a total of 15 8-week-old male wild-type C57BL/6 mice (SLAC Laboratory Animal, Shanghai, China). The animals were randomly divided into three groups: control group, GelMA hydrogel group and GA-GelMA hydrogel group. All surgical procedures were performed by two experienced orthopaedic surgeons. Briefly, the mice were anaesthetised with $2 \%$ pentobarbital and the hair on the left hind leg was shaved. The skin was disinfected by swabbing with alcohol. A lateral incision was made over the femur and the muscle was blunt dissected to expose the femur. The patella was then dislocated and a sterile 25-gauge needle was inserted into the femur shaft and retracted. The needle was positioned through the femur to stabilise the fracture section at the middle and lower segment that was created using a custommade three-point bender. Then, $3 \mu \mathrm{L}$ of GelMA with and without GA was pipetted over the fracture site and polymerised with UV irradiation for $15 \mathrm{~s}$. The control group received an equal volume of normal saline (NS). Finally, the muscle, patella and skin were sutured back into place.

\section{Radiographic analysis}

Animals were sacrificed at 1 month after surgery and the samples were collected and fixed in $4 \%$ paraformaldehyde for 72 hours at room temperature. For evaluation by micro-computed tomography (micro-CT), femoral samples were scanned for bone formation using a $\mu$ CT-100 Imaging System (Scanco Medical, Brüttisellen, Switzerland) with the following scan parameters: $70 \mathrm{kVp}$, reconstruction matrix of 1,024 and slice thickness of $14.8 \mu \mathrm{m}$ with an exposure time of $300 \mathrm{~ms}$. The trabecular number (Tb.N), trabecular thickness (Tb.Th), trabecular spacing ( $\mathrm{Tb} . \mathrm{Sp}$ ) and rabecular bone volume fraction (BV/TV were evaluated by 3D standard microstructural analysis (Bouxsein et al., 2010).

Histological evaluation

After micro-CT, all samples were decalcified using $10 \%$ ethylene diamine tetra acetic acid (EDTA; Sigma) in $0.1 \mathrm{M}$ PBS, changing the solution once a week for 6 weeks, before embedding in paraffin. Serial sections $3 \mu \mathrm{m}$ thick were cut and mounted on polylysine-coated slides, deparaffinised and then stained with hematoxylin and eosin (HE), Masson's trichrome, Safranin O and fast green separately on consecutive tissue sections based on our previous studies (Chen et al., 2017). Images were obtained using a microscope (Leica DM4000B; Leica, 
Wetzlar, Germany). We used a scoring scale for histological quantification based on cortical debridement and healing acceleration (Table 2). All evaluations were performed in a triple-blind manner.

Data and statistical analysis

All statistical analyses were performed using GraphPad Prism (version 8.0; GraphPad Software, San Diego, CA, USA). All experiments were conducted at least three times and the data are presented as means \pm SD. Differences between two groups were analysed by two-tailed Student'st test. For comparisons between more than two groups, one-way analysis of variance (ANOVA) followed by the Bonferroni post hoc test was used. In all analyses, $P<0.05$ was taken to indicate statistical significance.

\section{Results}

GA did not affect the viability or proliferation of hBMSCs

GA, consisting of two molecules glucuronic acid and one molecule of glycyrrhetinic acid, has an amphiphilic structure and carboxyl and hydroxyl groups (Fig. 1A). We performed CCK-8 assay to assess the effects of GA on the viability and proliferation of hBMSCs. GA showed no toxicity on hBMSCs at concentrations of 0-100 $\mu \mathrm{M}$ for 1, 2 or 3 days. No significant effect of GA on the proliferation of hBMSCs was observed (Fig. 1B).

GA increased the expression of osteo-specific genes and proteins and enhanced calcium deposition

To assess the role of GA in osteogenic differentiation of hBMSCs, osteo-specific gene and protein expression were examined by real time quantitative PCR, western blotting and immunofluorescence analyses.

The expression levels of osteo-related genes (RUNX2, COL1A1, OCN, Osterix and ALP) were markedly increased following GA treatment after 1 day of osteogenesis. The capacity of GA to promote osteogenesis varied at different concentrations. For RUNX2, COL1A1 and OCN, $100 \mu \mathrm{M}$ was the maximal concentration to produce a significant difference $(P<0.05)$, while $1 \mu \mathrm{M}$ was the maximal concentration for Osterix and ALP (Fig. 2A). Western blotting and immunofluorescence analyses indicated that the expression levels of osteo-related proteins (RUNX2 and COL1A1) were increased significantly compared to the control group on days 1 and 3 of osteogenesis. The effect of GA on osteogenesis was dose-dependent (Fig. 2B and 2C).

We investigated the influence of GA on late-stage calcium deposition and mineralised nodule formation during osteogenic differentiation by ARS. The results indicated that GA enhanced calcium deposition and mineralisation on day 14 in a dose-dependent manner (Fig. 2D). Early-stage mineralisation was conducted by ALP staining and activity assay on day 3. Similar to ARS, it showed that GA increased ALP activity (Fig. 2E).

GA promotes osteogenic differentiation of hBMSCs partly via the Wnt/ $\beta$-catenin signalling pathway

To explore the pathway underlying the regulation of osteogenic differentiation of hBMSCs by GA, we examined the most common signalling pathways involved in osteogenesis, including the Wnt/ $\beta$-catenin, NF- $x \beta$, PI3K-AKT and MAPK/ERK pathways. The expression of related proteins was investigated by western blotting (Fig. 3A) on day 3 of osteogenesis. Increased expression of active $\beta$-catenin was observed in the GA treatment groups, which was significant at $10 \mu \mathrm{M}$ and $100 \mu \mathrm{M}$ (but not $1 \mu \mathrm{M}$ ), while there were no significant differences in expression of total $\beta$-catenin between groups (Fig. 3B). Furthermore, there were no significant differences in components of the NF- $x \beta$, PI3K-AKT and MAPK/ERK pathways between groups (Fig. 3C-E).

Enhanced osteogenic differentiation of hBMSCs due to GA was partially reduced by the addition of Wnt/catenin signalling inhibitor

The Wnt/catenin signalling inhibitor XAV-939 was used to verify further the involvement of the Wnt/ $\beta$ catenin pathway in the induction of osteogenic differentiation by GA. After treatment with an appropriate concentration of XAV-939 (10 nM) that was confirmed to show a significant inhibitory effect (Fig. 4D), the increases in RUNX2 and COL1A1 expression induced by GA $(100 \mu \mathrm{M})$ were reversed as determined by 
western blotting (Fig. 4A-D). Immunofluorescence analysis also showed that inhibition of the Wnt/ $\beta$-catenin pathway partially reversed the increases in expression of RUNX2 and COL1A1 by GA (Fig. 4E). In addition, the enhanced mineralisation due to GA was suppressed after addition of XAV-939 as determined by ALP assay (Fig. 4E).

hBMSCs can survive in 2D and 3D GelMA scaffolds

We examined the structural characteristics of the surfaces of GelMA and GA/GelMA by SEM. GelMA and GA/GelMA both showed irregular elliptical pores that did not differ significantly in size between the two groups $(10.17 \pm 5.05 \mu \mathrm{m}$ and $10.22 \pm 5.57 \mu \mathrm{m}$, respectively, $P>0.5)$ (Fig. 5A-B). Furthermore, we investigated the survival of hBMSCs in GelMA. The results indicated that hBMSCs survived well and maintained their normal morphology in both 2D and 3D culture in GelMA (Fig. 5C-D).

GA -GelMA hydrogels accelerated bone healing in a mouse femoral fracture model

To further assess the effects of GA in vivo, we used GA-GelMA hydrogels (GA/GelMA) in a mouse femoral fracture model. Micro-CT showed that GA/GelMA significantly accelerated bone fracture healing compared to control and GelMA groups (Fig. 6B). One month after the operation, GA/GelMA significantly increased the BV/TV, Tb.N and Tb.Th, and significantly decreased Tb.Sp compared to the control group (Fig. 6C-F). Furthermore, GelMA alone without GA also showed superior BV/TV and Tb.Th values compared to the control group, although the GA/GelMA group showed significantly higher BV/TV and Tb.Th values and significantly lower Tb.Sp values (Fig. 6C-F). Histological evaluation, including HE, Masson's trichrome, Safranin $\mathrm{O}$ and fast green staining revealed that the GA/GelMA group had better fracture union and cortical bridge compared to the control group and GelMA group (Fig. 6G). The GA/GelMA group showed significantly increased histological evaluation scores compared to the control group. (Supplementary Figure 1).

\section{Discussion}

To our knowledge, this is the first study to show that GA promotes osteogenic differentiation of hBMSCs partly by modulating the Wnt/ $\beta$-catenin signalling pathway. We explored the influence of GA on the proliferation and osteogenic differentiation of hBMSCs. GA at concentrations from 1 to $100 \mu \mathrm{M}$ neither promoted nor suppressed the proliferation of hBMSCs in vitro, confirming its safety (Fig. 1). We found that RUNX2 and COL1A1 gene and protein expression levels were significantly increased after treatment with GA in a dose-dependent manner based on real time quantitative PCR, western blotting and immunofluorescence analyses (Fig. 2). Furthermore, other osteo-specific genes, such as Osterix, OCN and ALP, also showed significantly increased expression with GA treatment. The effects of GA on early and late mineralisation were also examined by ALP and ARS staining, which showed that GA enhanced the mineralisation of hBMSCs in a dose-dependent manner. Therefore, GA significantly promoted osteogenic differentiation and enhanced the mineralisation of hBMSCs. The in vivo study also indicated that GA can accelerate bone fracture healing in mice. It has been confirmed that GA can inhibit osteoclast formation and bone resorption function (Li et al., 2018; Yin et al., 2019). Our study provides further information regarding the pharmacological actions of GA in the field of orthopaedics. GA may have potential for the treatment of bone defects or non-union.

Wnt proteins are members of a family of secreted molecules that has been suggested to play roles throughout the healing process and in the promotion of osteoblast function (Krishnan et al., 2006). Wnt signals are transduced by a family of seven-transmembrane domain G protein-coupled receptors of the frizzled (FZD) family and a co-receptor of the arrow/LRP family (e.g., LRP5 and LRP6) or a Ryk or Ror transmembrane tyrosine kinase (Karner et al., 2017). Different Wnt proteins recognise their cognate receptors and activate at least three different intracellular signalling cascades: the canonical Wnt pathway (also known as the Wnt/ $\beta$-catenin pathway), the noncanonical Wnt pathway and the Wnt-calcium pathway (Hang et al., 2019). The best characterised of these is the Wnt/ $\beta$-catenin pathway. Wnt proteins activate the FZD/LRP5 or FZD/LRP6 receptor complexes and thus stabilise $\beta$-catenin in the cytoplasm Subsequently, $\beta$-catenin enters the nucleus and regulates the expression of target genes. In our study, we detected higher levels of expression of active $\beta$-catenin after GA treatment during osteogenesis. Meanwhile, lower active $\beta$-catenin and osteogenic 
specific protein (RUNX2 and COL1A1) expression levels were observed after treatment with the Wnt/ $\beta$ catenin inhibitor, XAV-939, compared to GA. These observations indicate that GA promoted the osteogenic differentiation of hMSCs partly by modulating the Wnt/ $\beta$-catenin signalling pathway.

Tissue engineering and regenerative medicine have emerged as promising strategies for bone reconstitution (Henkel et al., 2013). Various tissue-engineered scaffolds have been fabricated and applied in bone tissue engineering (BTE). Scaffold characteristics that can be modulated, improved or changed to make it more suitable for BTE applications must possess suitable biological and structural features, such as biocompatibility, no toxicity, biodegradability, bioactivity, osteoinductivity and osteoconductivity, as well as osteogenic features and mechanical properties (Roseti et al., 2017). Several different scaffolds have been developed, made of both natural and synthetic materials. Natural materials, such as collagen, silk fibroin and chitosan, show good biocompatibility and allow cell attachment and growth. However, natural materials have poor mechanical strength, making it difficult to control degradation time. Synthetic materials, including hydroxyapatite (HA), polylactic acid (PLA) and GelMA, have been used as scaffolds. However, HA does not have osteoinductive properties and PLA has weak cell attachment capacity and poor mechanical strength. GelMA hydrogels have been used extensively for various biomedical applications requiring in vivo mimicry and 3D cell culture due to their suitable biological properties and tuneable physical characteristics (Yue et al., 2015). GelMA has RGD sites (Arg-Gly-Asp) for cell attachment, matrix metalloproteinase recognition sequences for biodegradability, excellent biocompatibility and tuneable physical properties (Choi et al., 2019). hBMSCs showed good survival in 2D and 3D culture in GelMA. Porosity and pore size are very important parameters in designing scaffolds for bone regeneration. SEM indicated that GelMA and GA/GelMA are porous, which contributes to osteogenesis. Ning et al. (Ning et al., 2019) reported that injectable photo-crosslinked GelMA hydrogel promoted the healing of bone defects in rats. We designed a GA-delivered GelMA hydrogel, which was used in a mouse femoral fracture model, and the results of micro-CT and histological evaluation indicated that it had a superior effect on bone-fracture healing.

Several animal models have been used to study fracture non-union or delayed union (Cappellari et al., 2014; Chu et al., 2009; Lozada-Gallegos et al., 2013; Shimizu et al., 2015). In recent years, fracture research has focused on rats and mice (Garcia et al., 2013). However, the definitions of fracture non-union and delayed union are inconsistent (Garcia et al., 2013). Some researchers chose transverse osteotomy with additional periosteum resection to study fracture non-union or impaired fracture healing in mouse models. This approach may simulate open fractures with severe soft tissue injury, which are associated with a high incidence of non-union in clinical cases.

Our study had some limitations. First, GA modulated the Wnt/ $\beta$-catenin signalling pathway to regulate osteogenic differentiation, and it is also likely involved in the activation of other signalling pathways. In addition to the canonical actions, non-canonical signals were not explored. Second, the underlying mechanisms, such as the cell-surface receptors that mediate signal transmission, were not clarified. Third, we investigated only intramembranous ossification in our study, and further studies are required to determine the impact of GA on the processes of endochondral ossification.

\section{Conclusions}

In conclusion, our study showed that GA promotes the osteogenic differentiation of hMSCs by modulating the Wnt/ $\beta$-catenin signalling pathway. GA-containing GelMA hydrogel effectively accelerated bone-fracture healing.

\section{Disclaimer:}

Funding was provided by the Zhejiang Provincial Natural Science Foundation of China (No. LY18H060003, No. LY16H060003 and No. LQ18H050005), the National Natural Science Foundation of China (No.8187090251, No.81871759, No.81672147).

\section{References}


Ankrum JA, Ong JF, \& Karp JM (2014). Mesenchymal stem cells: immune evasive, not immune privileged. Nature biotechnology 32:252-260.

Atasoy-Zeybek A, \& Kose GT (2018). Gene Therapy Strategies in Bone Tissue Engineering and Current Clinical Applications. Advances in experimental medicine and biology 1119: 85-101.

Begam H, Nandi SK, Kundu B, \& Chanda A (2017). Strategies for delivering bone morphogenetic protein for bone healing. Materials science \& engineering C, Materials for biological applications 70: 856-869.

Bhattacharjee S, Bhattacharjee A, Majumder S, Majumdar SB, \& Majumdar S (2012). Glycyrrhizic acid suppresses Cox-2-mediated anti-inflammatory responses during Leishmania donovani infection. J Antimicrob Chemother 67: 1905-1914.

Bouxsein ML, Boyd SK, Christiansen BA, Guldberg RE, Jepsen KJ, \& Muller R (2010). Guidelines for assessment of bone microstructure in rodents using micro-computed tomography. Journal of bone and mineral research : the official journal of the American Society for Bone and Mineral Research 25: 1468-1486.

Cappellari F, Piras L, Panichi E, Ferretti A, \& Peirone B (2014). Treatment of antebrachial and crural septic nonunion fractures in dogs using circular external skeletal fixation: a retrospective study. Veterinary and comparative orthopaedics and traumatology : VCOT 27: 297-305.

Chen EEM, Zhang W, Ye CCY, Gao X, Jiang LLJ, Zhao TTF, et al.(2017). Knockdown of SIRT7 enhances the osteogenic differentiation of human bone marrow mesenchymal stem cells partly via activation of the Wnt/beta-catenin signaling pathway. Cell Death Dis 8: e3042.

Choi BY, Chalisserry EP, Kim MH, Kang HW, Choi IW, \& Nam SY (2019). The Influence of Astaxanthin on the Proliferation of Adipose-derived Mesenchymal Stem Cells in Gelatin-Methacryloyl (GelMA) Hydrogels. Materials (Basel, Switzerland) 12.

Chu T, Liu Y, Wang Z, Zhu P, Jiao W, Wen J, et al. (2009). Sustained vascular endothelial growth factor blockade by antivascular endothelial growth factor antibodies results in nonunion in the process of fracture healing in rabbits. The Journal of trauma 66:1180-1183.

Coutu DL, Kokkaliaris KD, Kunz L, \& Schroeder T (2017). Three-dimensional map of nonhematopoietic bone and bone-marrow cells and molecules. Nature biotechnology 35: 1202-1210.

Dekker TJ, White P, \& Adams SB (2017). Efficacy of a Cellular Bone Allograft for Foot and Ankle Arthrodesis and Revision Nonunion Procedures. Foot Ankle Int 38: 277-282.

Dimitriou R, Jones E, McGonagle D, \& Giannoudis PV (2011). Bone regeneration: current concepts and future directions. BMC Med 9: 66.

Fassbender M, Minkwitz S, Strobel C, Schmidmaier G, \& Wildemann B (2014). Stimulation of bone healing by sustained bone morphogenetic protein 2 (BMP-2) delivery. Int J Mol Sci 15: 8539-8552.

Fazzalari NL (2011). Bone fracture and bone fracture repair. Osteoporosis international : a journal established as result of cooperation between the European Foundation for Osteoporosis and the National Osteoporosis Foundation of the USA 22: 2003-2006.

Feng X, Ding L, \& Qiu F (2015). Potential drug interactions associated with glycyrrhizin and glycyrrhetinic acid. Drug Metab Rev 47:229-238.

Garcia P, Histing T, Holstein JH, Klein M, Laschke MW, Matthys R, et al. (2013). Rodent animal models of delayed bone healing and non-union formation: a comprehensive review. Eur Cell Mater 26:1-12; discussion $12-14$.

Giannoudis PV, Jones E, \& Einhorn TA (2011). Fracture healing and bone repair. Injury 42: 549-550.

Gibon E, Lu L, \& Goodman SB (2016). Aging, inflammation, stem cells, and bone healing. Stem Cell Res Ther 7: 44. 
Hang K, Ye C, Xu J, Chen E, Wang C, Zhang W, et al. (2019). Apelin enhances the osteogenic differentiation of human bone marrow mesenchymal stem cells partly through Wnt/beta-catenin signaling pathway. Stem Cell Res Ther 10: 189.

Henkel J, Woodruff MA, Epari DR, Steck R, Glatt V, Dickinson IC, et al. (2013). Bone Regeneration Based on Tissue Engineering Conceptions - A 21st Century Perspective. Bone Res 1: 216-248.

Iwatani S, Shono A, Yoshida M, Yamana K, Thwin KKM, Kuroda J, et al. (2017). Involvement of WNT Signaling in the Regulation of Gestational Age-Dependent Umbilical Cord-Derived Mesenchymal Stem Cell Proliferation. Stem Cells Int 2017: 8749751.

Johnson CT, Wroe JA, Agarwal R, Martin KE, Guldberg RE, Donlan RM, et al. (2018). Hydrogel delivery of lysostaphin eliminates orthopedic implant infection by Staphylococcus aureus and supports fracture healing. Proc Natl Acad Sci U S A 115: E4960-E4969.

Karner CM, \& Long F (2017). Wnt signaling and cellular metabolism in osteoblasts. Cell Mol Life Sci 74: $1649-1657$.

Krishnan V, Bryant HU, \& Macdougald OA (2006). Regulation of bone mass by Wnt signaling. J Clin Invest 116: 1202-1209.

Li Z, Chen C, Zhu X, Li Y, Yu R, \& Xu W (2018). Glycyrrhizin Suppresses RANKL-Induced Osteoclastogenesis and Oxidative Stress Through Inhibiting NF-kappaB and MAPK and Activating AMPK/Nrf2. Calcif Tissue Int 103: 324-337.

Livak KJ, \& Schmittgen TD (2001). Analysis of relative gene expression data using real-time quantitative PCR and the 2(-Delta Delta C(T)) Method. Methods 25: 402-408.

Lozada-Gallegos AR, Letechipia-Moreno J, Palma-Lara I, Montero AA, Rodriguez G, Castro-Munozledo F, et al. (2013). Development of a bone nonunion in a noncritical segmental tibia defect model in sheep utilizing interlocking nail as an internal fixation system. J Surg Res 183: 620-628.

Murata K, Ito H, Yoshitomi H, Yamamoto K, Fukuda A, Yoshikawa J, et al. (2014). Inhibition of miR-92a enhances fracture healing via promoting angiogenesis in a model of stabilized fracture in young mice. Journal of bone and mineral research : the official journal of the American Society for Bone and Mineral Research 29: $316-326$.

Ning Z, Tan B, Chen B, Lau DSA, Wong TM, Sun T, et al. (2019). Precisely Controlled Delivery of Abaloparatide through Injectable Hydrogel to Promote Bone Regeneration. Macromolecular bioscience 19: e1900020.

Park JS, Suryaprakash S, Lao YH, \& Leong KW (2015). Engineering mesenchymal stem cells for regenerative medicine and drug delivery. Methods 84: 3-16.

Roseti L, Parisi V, Petretta M, Cavallo C, Desando G, Bartolotti I, et al. (2017). Scaffolds for Bone Tissue Engineering: State of the art and new perspectives. Materials science \& engineering C, Materials for biological applications 78: 1246-1262.

Shapiro G, Lieber R, Gazit D, \& Pelled G (2018). Recent Advances and Future of Gene Therapy for Bone Regeneration. Curr Osteoporos Rep 16: 504-511.

Shimizu T, Akahane M, Morita Y, Omokawa S, Nakano K, Kira T, et al. (2015). The regeneration and augmentation of bone with injectable osteogenic cell sheet in a rat critical fracture healing model. Injury 46: $1457-1464$.

Su X, Wu L, Hu M, Dong W, Xu M, \& Zhang P (2017). Glycyrrhizic acid: A promising carrier material for anticancer therapy. Biomedicine \& pharmacotherapy = Biomedecine \& pharmacotherapie 95: 670-678. 
Sun ZG, Zhao TT, Lu N, Yang YA, \& Zhu HL (2019). Research Progress of Glycyrrhizic Acid on Antiviral Activity. Mini Rev Med Chem 19:826-832.

Wang CY, Kao TC, Lo WH, \& Yen GC (2011). Glycyrrhizic acid and 18beta-glycyrrhetinic acid modulate lipopolysaccharide-induced inflammatory response by suppression of NF-kappaB through PI3K p110delta and p110gamma inhibitions. J Agric Food Chem 59: 7726-7733.

Yin Z, Zhu W, Wu Q, Zhang Q, Guo S, Liu T, et al. (2019). Glycyrrhizic acid suppresses osteoclast differentiation and postmenopausal osteoporosis by modulating the NF-kappaB, ERK, and JNK signaling pathways. Eur J Pharmacol 859: 172550.

Yue K, Trujillo-de Santiago G, Alvarez MM, Tamayol A, Annabi N, \& Khademhosseini A (2015). Synthesis, properties, and biomedical applications of gelatin methacryloyl (GelMA) hydrogels. Biomaterials 73: 254271.

Zhang W, Chen E, Chen M, Ye C, Qi Y, Ding Q, et al. (2018). IGFBP7 regulates the osteogenic differentiation of bone marrow-derived mesenchymal stem cells via Wnt/beta-catenin signaling pathway. FASEB J 32: $2280-2291$.

Zura R, Xiong Z, Einhorn T, Watson JT, Ostrum RF, Prayson MJ, et al. (2016). Epidemiology of Fracture Nonunion in 18 Human Bones. JAMA Surg 151: e162775.

TABLE I Sequences of primers for real-time quantitative PCR analysis

\begin{tabular}{lll}
\hline Gene & Forward Primer & Reverse Primer \\
\hline COL1A1 & CAGATCACGTCATCGCACAAC & GAGGGCCAAGACGAAGACATC \\
RUNX2 & TGGTTACTGTCATGGCGGGTA & TCTCAGATCGTTGAACCTTGCTA \\
OCN & CACTCCTCGCCCTATTGGC & CCCTCCTGCTTGGACACAAAG \\
Osterix & AGCCCATTAGTGCTTGTAAAGG & CCTCTGCGGGACTCAACAAC \\
ALP & ACCACCACGAGAGTGAACCA & CGTTGTCTGAGTACCAGTCCC \\
GAPDH & GGAGCGAGATCCCTCCAAAAT & GGCTGTTGTCATACTTCTCATGG \\
\hline
\end{tabular}

TABLE II Histological evaluation scoring scale of fracture healing

\begin{tabular}{ll}
\hline Scores & Histological evaluation sites \\
\hline 0 & No bridging, no callus formation \\
1 & No bridging, initiation of a small amount callus \\
2 & No bridging, obvious initial callus formation near fracture \\
3 & No bridging, marked callus formation near and around fracture site \\
4 & Bridging of at least one of the cortices, marked callus formation near and around fracture site \\
5 & Bridging of at least one of the cortices, marked and complete callus formation around fracture site \\
6 & Bridging of both cortices, and/or some resolution of the callus \\
7 & Clear bridging of both cortices and resolution of the callus \\
\hline
\end{tabular}

\section{Figure legends}

Fig. 1. GA had no effect on the viability or proliferation of hBMSCs. (A) The chemical structure of GA. (B) The proliferation of hMSCs was measured by CCK- 8 assay after addition of different concentrations of GA $(0-100 \mu \mathrm{M})$ for 1,2 or 3 days.

Fig. 2. GA increased the expression levels of osteo-specific genes and proteins and enhanced calcium deposition. (A) Relative mRNA expression of osteo-related genes (RUNX2, COL1A1, Osterix, OCN and 
ALP) measured by real time quantitative PCR after 1 day of osteogenesis. The mRNA expression levels were normalised relative to that of GAPDH RNA. (B) Relative protein expression levels of osteo-related protein (RUNX2 and COL1A1) were measured by western blotting after 1 and 3 days of osteogenesis. The protein expression levels were normalised relative to that of GAPDH protein. (C) Immunofluorescence staining for COL1A1 and RUNX2 protein after 1 day of osteogenesis. Scale bars, $100 \mu \mathrm{m}$. (D) Mineralisation was measured by ARS staining and quantitative assay after 14 days of osteogenesis. Scar bar, $500 \mu \mathrm{m}$. (E) ALP staining and quantitative assay on day 3 of osteogenic differentiation. Scar bar, $500 \mu \mathrm{m}$. All data are expressed as the means $\pm \mathrm{SD}$. Reactions were performed in triplicate. ${ }^{*} \mathrm{P}<0.05,{ }^{* *} \mathrm{P}<0.01$ compared to the control group.

Fig. 3. GA promotes osteogenic differentiation of hMSCs partly via the Wnt/ $\beta$-catenin signalling pathway. (A) Relative protein expression levels of components of different signalling pathways were assessed by western blotting. hMSCs were incubated with different concentrations of GA during osteogenic differentiation on day 3. The protein expression levels were normalised relative to that of GAPDH protein. (B-E) Relative quantitative analysis of western blotting results for active $\beta$-catenin ( $\beta$-catenin) and total $\beta$-catenin ( $\mathrm{t} \beta$ catenin), p-p65 and t-p65, p-AKT and t-AKT, p-ERK and t-ERK. All tests were performed in triplicate. Data are expressed as means $\pm \mathrm{SD} .{ }^{* *} \mathrm{P}<0.01$ compared to the control group.

Fig. 4. Enhanced osteogenic differentiation of hMSCs due to GA was partially reduced by the addition of Wnt/catenin signalling inhibitor. (A) Inhibition of the Wnt/catenin signalling pathway partially reversed the increases in RUNX2 and COL1A1 protein expression levels after addition of XAV-939 for 3 days. The protein expression levels were normalised relative to that of GAPDH protein. (B-D) Relative quantitative analysis of the western blotting results for active $\beta$-catenin, RUNX2 and COL1A1 protein. (E) Immunofluorescence staining after 3 days of osteogenesis. Scale bars, $100 \mu \mathrm{m}$. (F) ALP revealed decreased mineralisation in the GA/XAV-939 group compared to the GA group. Scar bar, $500 \mu \mathrm{m}$. Data are expressed as means \pm SD. * $\mathrm{P}<0.05$ and ${ }^{* *} \mathrm{P}<0.01$ between the two groups.

Fig. 5. hBMSCs survived in 2D and 3D culture in GelMA scaffolds. (A-B) The structural features on the surfaces of GelMA and GA/GelMA. Scale bars, $10 \mu \mathrm{m}$. (C-D) Culture of GFP-hBMSCs in 2D and 3D GelMA scaffolds. Scale bars, $100 \mu \mathrm{m}$.

Fig. 6. GA/GelMA hydrogels accelerated bone healing in a mouse femoral fracture model. (A) Schematic diagram of mouse femoral fracture model. (A) Micro-CT analysis of bone fracture healing. (B-E) Bone volume and trabecular thickness were analysed by micro-CT. All experiments were performed in triplicate. (F) Histological evaluation, including HE, Masson's trichrome, Safranin O and fast green staining, of mouse femoral fracture area at 1 month after surgery. Scar bar, $500 \mu \mathrm{m}$. Data are expressed as means $\pm \mathrm{SD}$. ${ }^{*} \mathrm{P}$ $<0.05$ and ${ }^{* *} \mathrm{P}<0.01$ between two groups.

Supplementary figure 1 The GA/GelMA group had a significant increase in the histological evaluation score. Dates were expressed as mean $\pm \mathrm{SD} .{ }^{* *} \mathrm{P}<0.01$ indicate the significant difference between two groups.

A

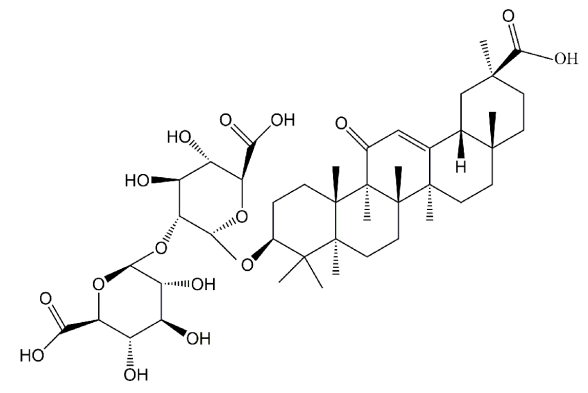

B

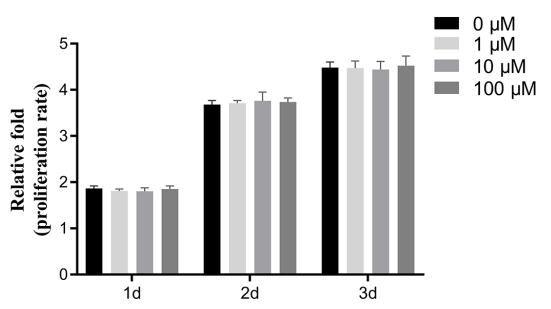



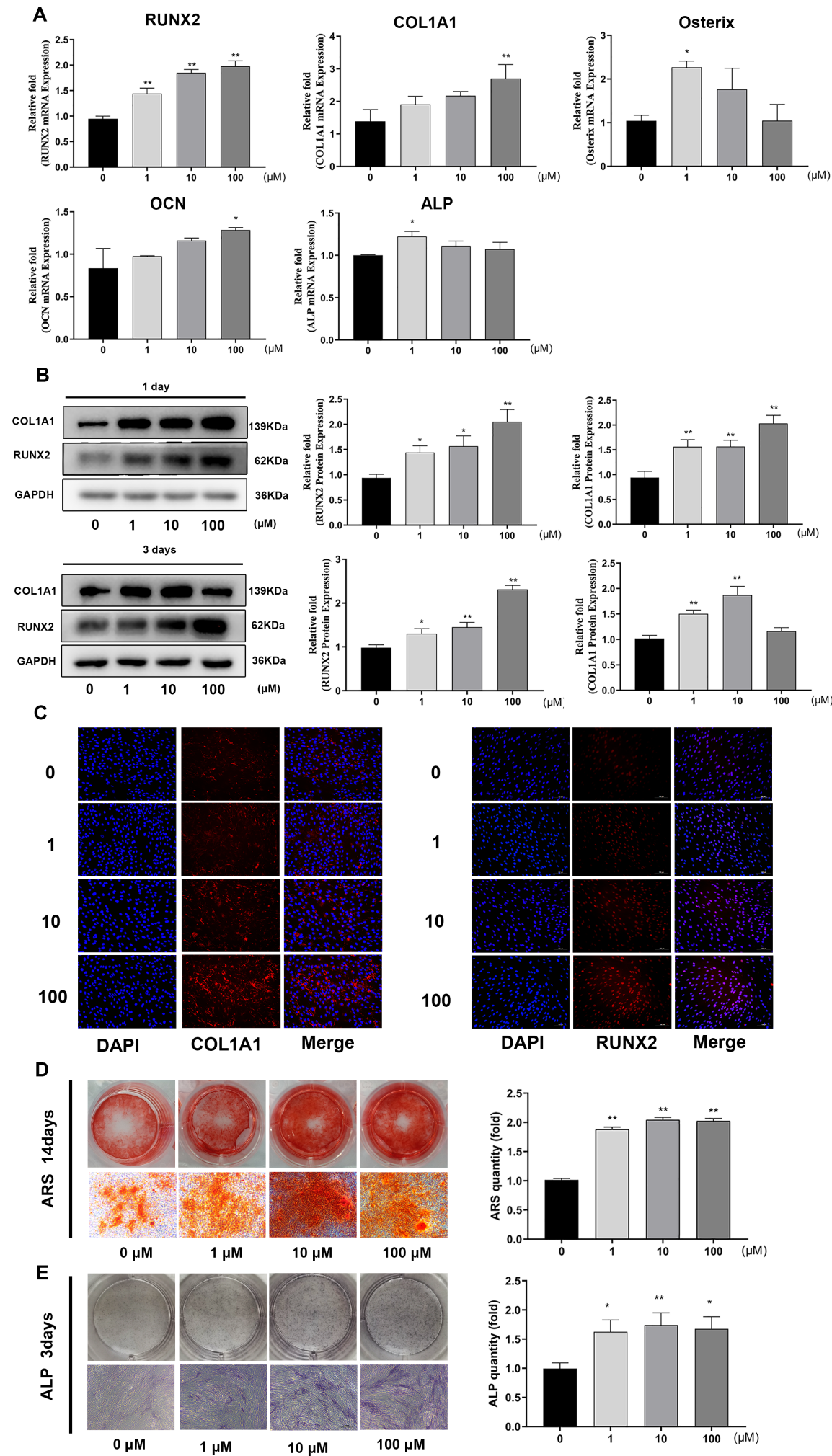

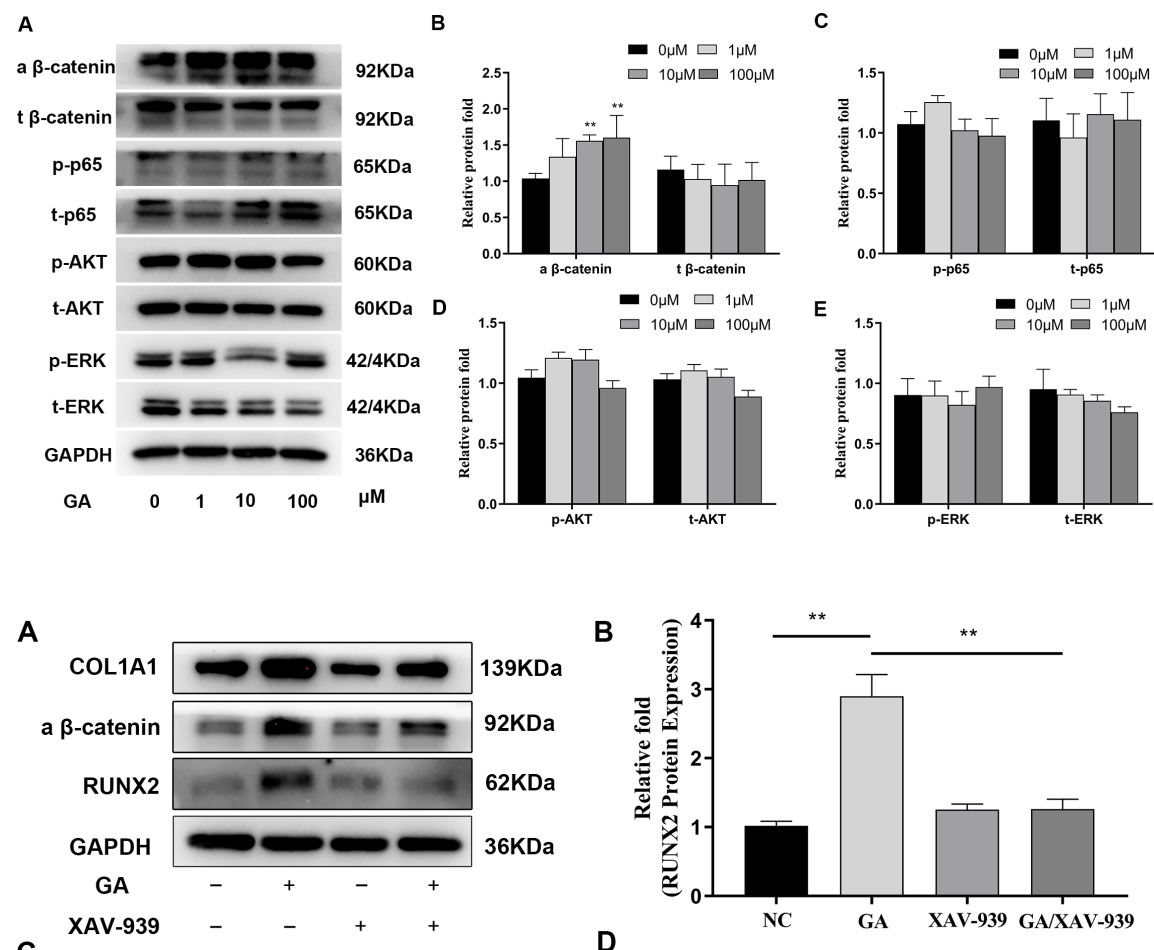

C
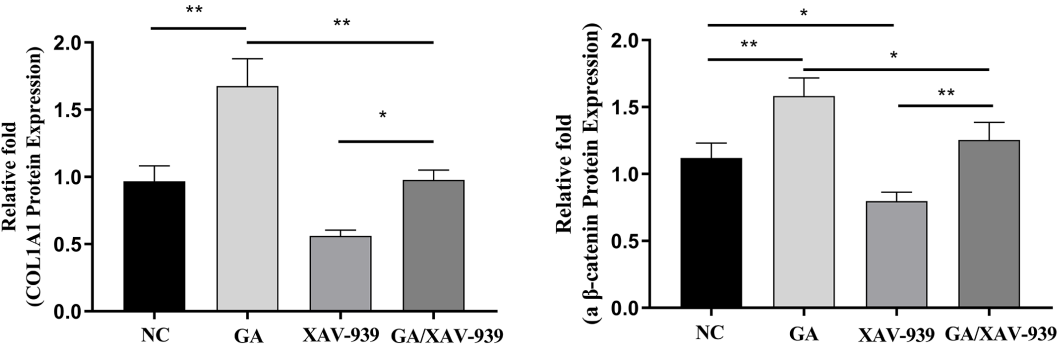

E
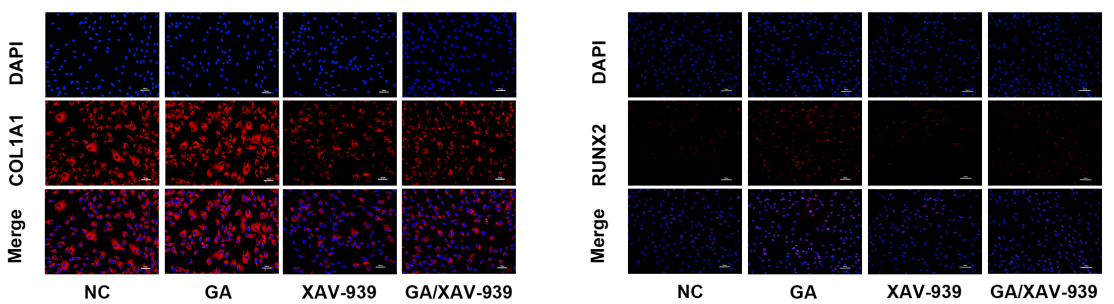

F
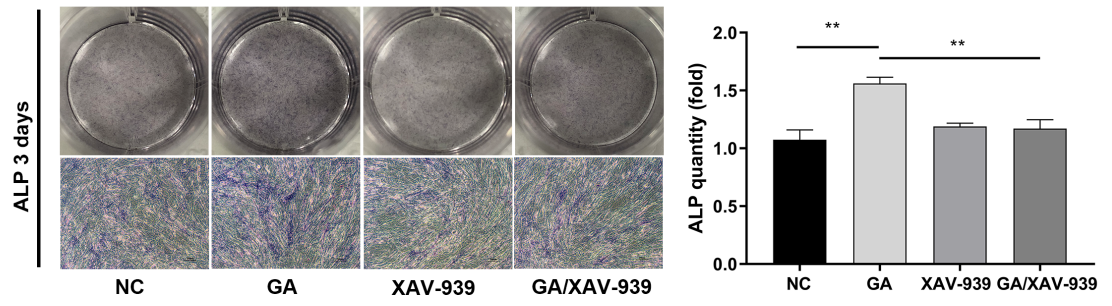


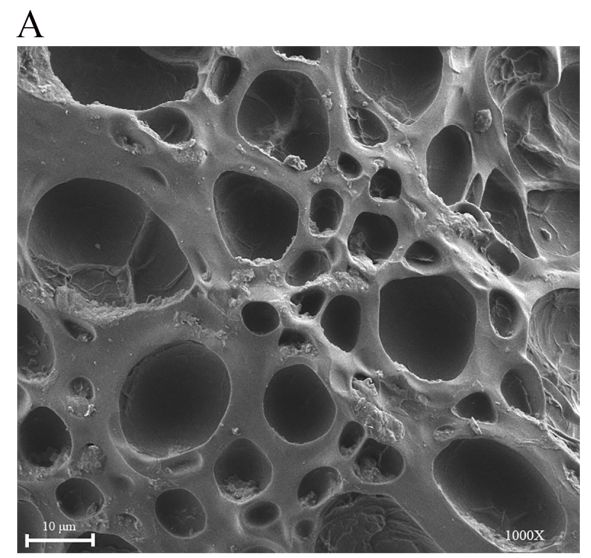

GelMA

C

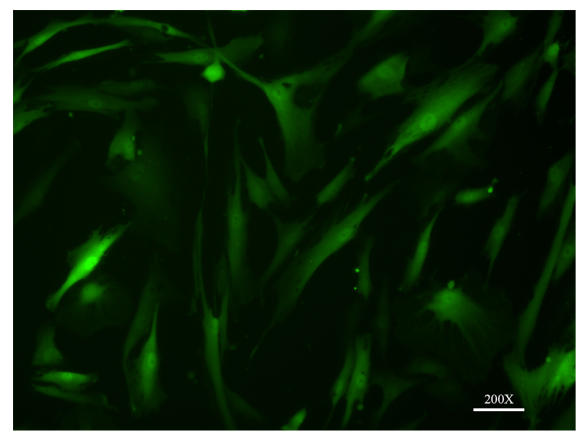

hBMSCs 2D culture
B

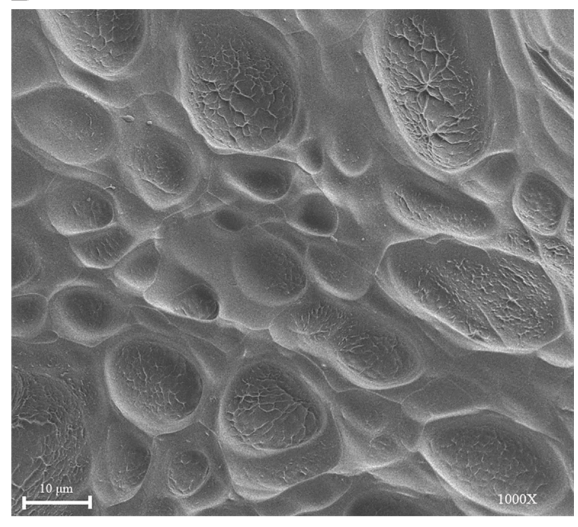

GA/GelMA

D

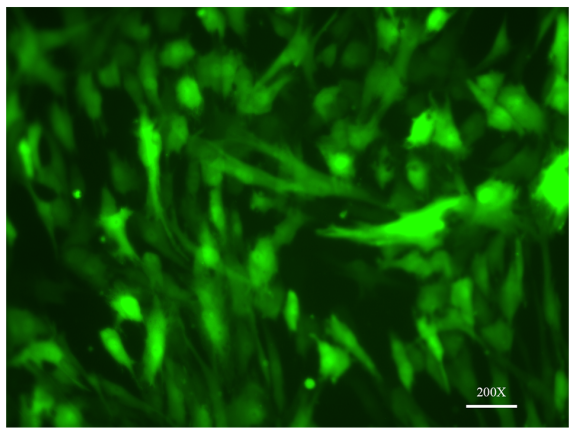

hBMSCs 3D culture 
A

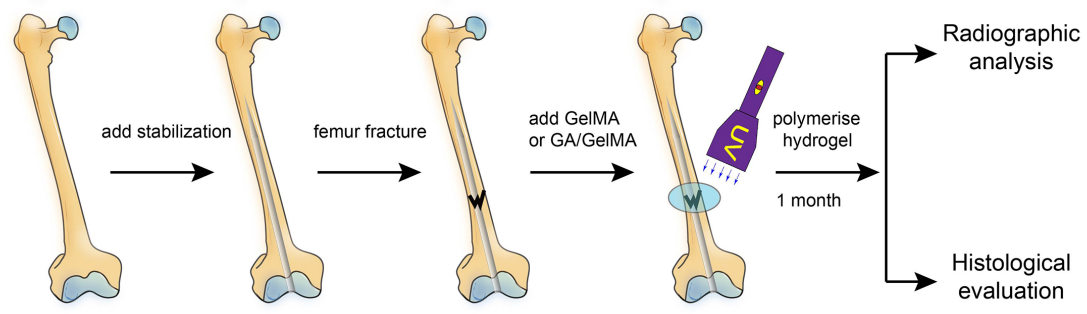

B

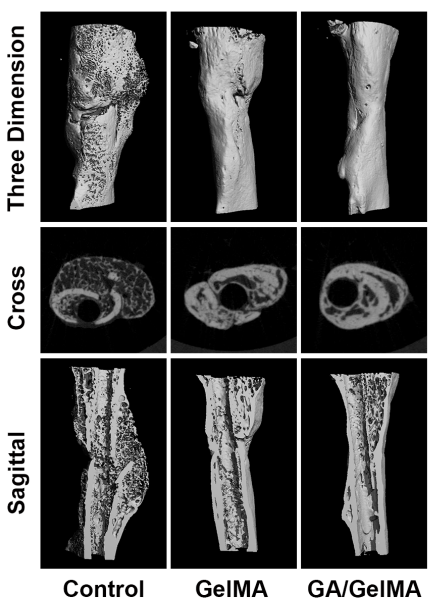

G
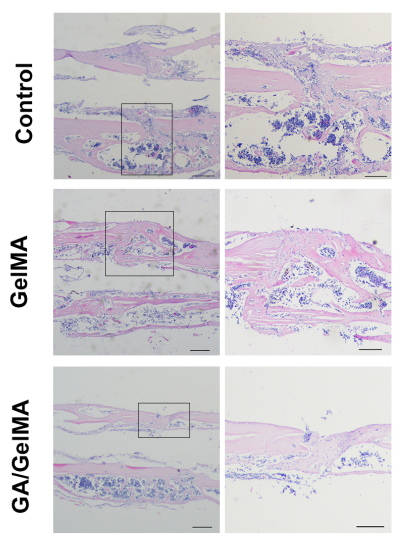

HE
C

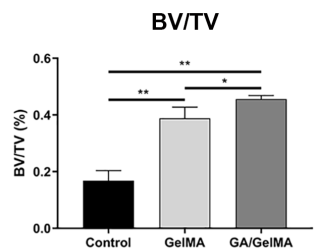

E
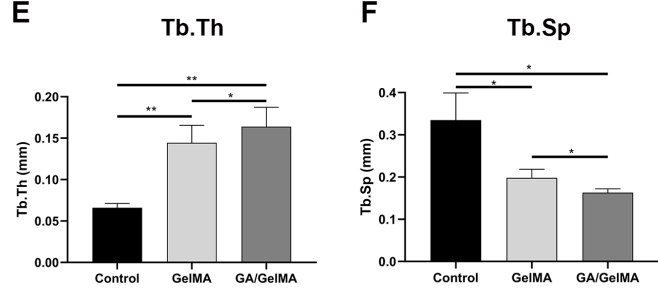

F

Tb.N
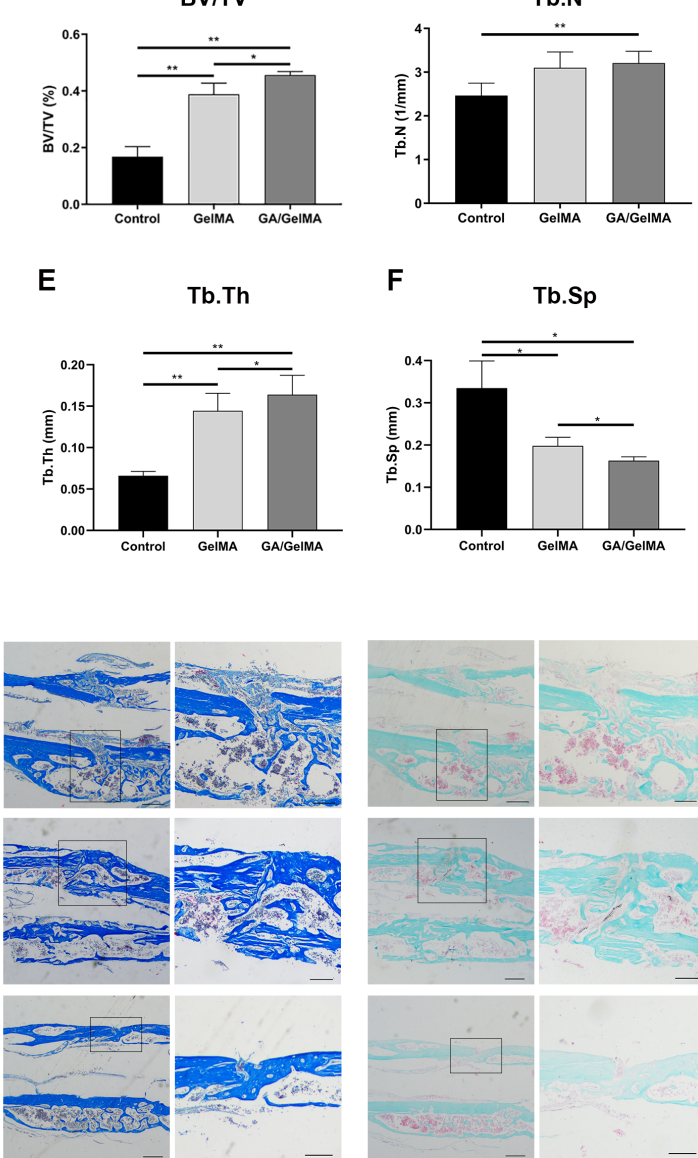

Masson
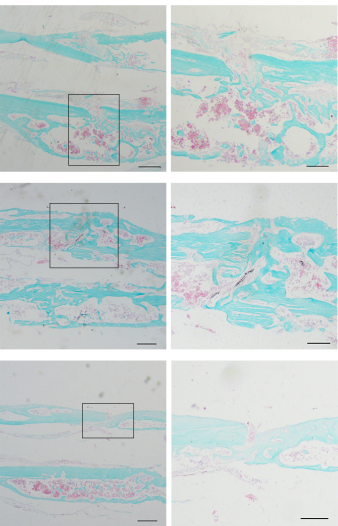

Safranin O 


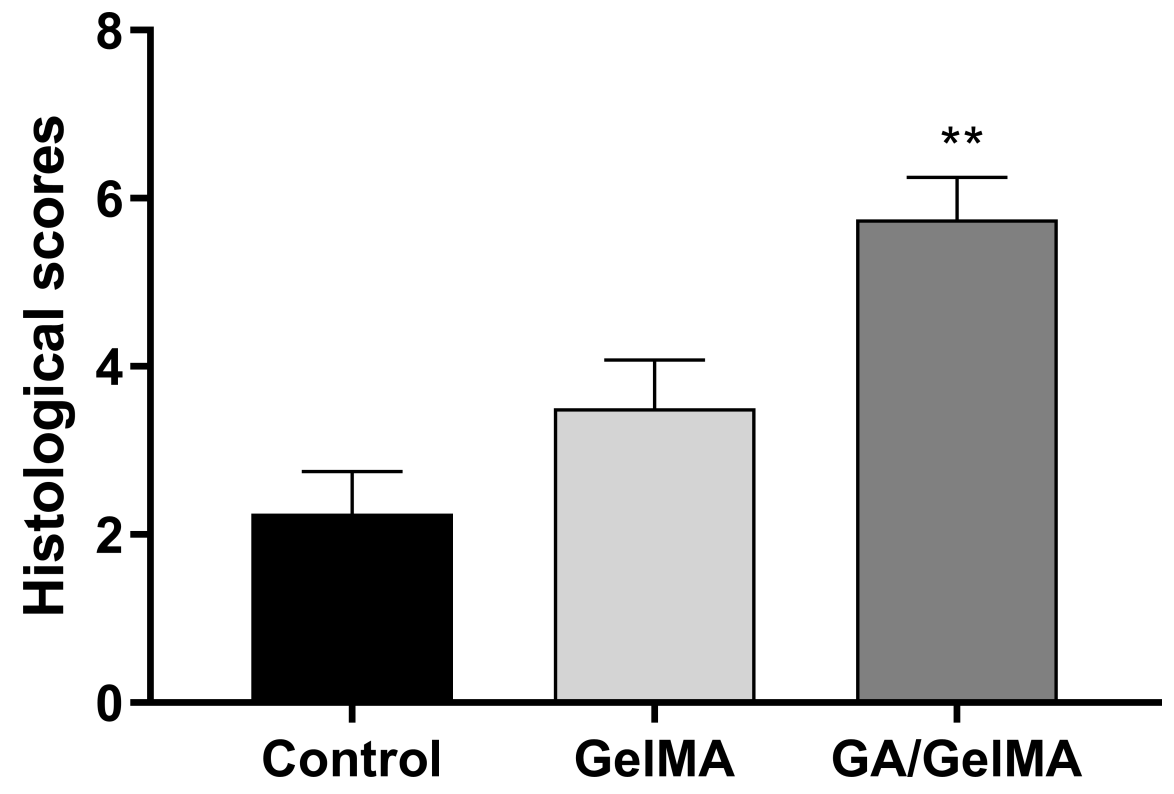

